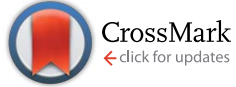

Cite this: RSC Adv., 2015, 5, 57425
Received 23rd May 2015

Accepted 25th June 2015

DOI: $10.1039 / \mathrm{c} 5 \mathrm{ra09704e}$

www.rsc.org/advances

\title{
Controlling the cooperative self-assembly of graphene oxide quantum dots in aqueous solutions $\uparrow$
}

\begin{abstract}
Salman Hassanzadeh, ${ }^{*}$ Karin H. Adolfsson and Minna Hakkarainen
Fascinating 3D cooperative self-assembly behavior was observed for 2D graphene oxide quantum dots (GOQDs) in dilute and semi dilute aqueous solutions. In addition the optical properties could be tuned by controlling the supramolecular structures. While the electrostatic interactions between the charged single sheets were assigned as the main secondary interactions that were responsible for the supramolecular fine structures, the concentration, temperature, salt concentration and $\mathrm{pH}$ could tune the repulsive/attractive forces and the molecular binding between the GOQD sheets. The morphological studies combined with UV-Vis and fluorescence evaluations proved that after a slow nucleation step, elongation preceded radially by $\mathrm{H}$-aggregate self-association of the GOQD monomers, forming the final porous spheres by radial growth of rods. The quenching properties of the self-associated-assembled GOQDs together with the excitation wavelengths of the GOQD solutions enabled tuning of the fluorescence intensity and color of the final solutions, which could be utilized for e.g. bioimaging and smart spectroscopy.
\end{abstract}

\section{Introduction}

Honeycomb lattice graphene and chemically modified graphene, such as graphene oxide (GO), have revitalized the twodimensional (2D) carbon structure research in the past decade..$^{1-6}$ The unique structure of monolayered graphene provides extraordinary electronic properties and excellent thermal and electrical conductivity. ${ }^{7-11}$ Intrinsic graphene is insoluble, intractable and decomposes before melting, while GO as its chemical derivative displays fully different properties. ${ }^{12,13}$ The core of GO consists of small $\mathrm{sp}^{2}$ hybridized 2D carbon domains that are surrounded by $\mathrm{sp}^{3}$ hybridized carbon functionalized with carboxyl, epoxy and hydroxyl groups providing solubility in $\mathrm{H}_{2} \mathrm{O} .^{14}$ The specific structural-properties of GO hold great promise for diverse technologies, including nanoelectronic and photoelectronic devices, ${ }^{15}$ energy storage materials, ${ }^{16}$ polymeric nanocomposites, ${ }^{17}$ catalysis $^{18}$ and biomedicine. ${ }^{19}$ Water solubility, biocompatibility and fluorescence properties make GO a highly interesting material for biomedical applications. ${ }^{20,21}$ The non-toxic nature of GO, its excitation wavelengths in the visible area, intrinsic fluorescence

Department of Fibre and Polymer Technology, School of Chemical Science and Engineering, KTH Royal Institute of Technology, Stockholm, Sweden. E-mail: salmanha@kth.se

$\dagger$ Electronic supplementary information (ESI) available: DSC and TGA analyses, RAMAN spectroscopy, particle sizes of GOQD solutions, zeta potentials $(\zeta)$ of GOQD solutions, TEM images, UV-Vis transmittance, AFM images and size analysis in the dried state and fluorescence spectroscopy. See DOI: 10.1039/c5ra09704e brightness as well as its photo-stability provide advantages over the semiconducting small particle alternatives (such as cadmium) in cell imaging applications. ${ }^{22}$

Depending on the size, GO can sometimes be referred to as graphene oxide quantum dots (GOQDs) meaning very small functionalized nanoparticles of less than $100 \mathrm{~nm}$ (diameter) in solution. ${ }^{23}$ GOQDs and other members of the carbon quantum dot (CQD) family display attractive optical and electro-optical properties due to their quantum confinement and edge effects. ${ }^{24}$ GOQDs exhibit similar to their larger relatives, GOs, amphiphilic properties with hydrophilic edges and more hydrophobic central basal planes. ${ }^{12}$ Due to this structure, they can disperse as colloids in aqueous medium without any surfactants or stabilizing polymers. ${ }^{25,26}$ At the same time, the provided dimensional features, functional groups with the coexistence of oxidized and aromatic moieties and the resulting secondary interactions such as electrostatic, $\pi-\pi$, hydrogen bonding and/or hydrophobic interactions make the GOQDs attractive building blocks for the construction of supramolecular architectures. ${ }^{25,27}$ Their self-recognition capability at molecular level leads to potential self-assembly and formation of different 3D structures at a macroscopic level such as ultrathin films, ${ }^{28}$ paper-like materials ${ }^{29}$ and 3D networks. ${ }^{30}$ The smartness of the formed 3D supramolecular structures originates from the tunable secondary interactions controlled by physical parameters, as temperature, $\mathrm{pH}$ and ionic strength of the medium..$^{31,32}$ Due to the simultaneous presence of different types of secondary interactions in GOQDs, the interplay of several different interactions causes negative or positive 
cooperativity of the molecular units depending on if the coupling of the interactions is favorable or disfavorable. ${ }^{33}$ There are two main synthesis strategies for production of GO: (a) topdown synthesis such as the modified and unmodified hummer methods using mainly graphite as starting material ${ }^{34}$ and (b) bottom-up synthesis using small molecules as starting materials. ${ }^{35,36}$ The GOQDs in the current work were synthesized from a novel raw material by utilizing as an initial step our newly developed microwave assisted process. ${ }^{37,38}$ As a final step the amorphous carbon nanospheres $(\mathrm{CN})$ from the microwave process, build-up of disordered-small graphitic flakes, were then oxidized to graphene oxide. The suggested synthesis strategy possess advantages such as: renewable cheap starting material (cellulose), green and easy synthesis method leading to fine nanometric GO structures instead of the more common larger graphene oxide sheets.

Despite some current insights concerning the assembly of micrometer sized regular GO in the concentrated regime e.g. hydrogels, ${ }^{31}$ solid phase ${ }^{39,40}$ and the liquid crystal behavior of $\mathrm{GO},{ }^{41}$ the cooperative self-assembly of small GOQDs in the dilute and semi-dilute regime has not been investigated. We hypothesized that controllable supramolecular structures and tunable morphologies for the self-assembling systems could be achieved by adjusting the $\mathrm{pH}$, ionic strength and concentration of the GOQD solutions. Control over the GOQDs supramolecular cooperativity behavior from single molecules to supramolecularly self-assembled structures in the solution is a stepping stone towards introducing smart and fine structures capable of accomplishing modern material missions in target applications. ${ }^{2}$ Our results provide unique insights into the 3D self-assembly mechanisms, main operative secondary interactions, controllable fluorescence properties of GOQDs and the possibilities to tune the cooperativity of the synthesized GOQDs in dilute and semi dilute regimes. This could finally enable controllable, low cost and scalable self-assembling GOQD systems forming 3D carbonous structures with possible applications ranging from electronics and clean energy devices to sensors and novel biomedical materials. ${ }^{\mathbf{4 2 , 4 3}}$

\section{Experimental}

\section{Materials}

Sulfuric acid $\left(\mathrm{H}_{2} \mathrm{SO}_{4}, 95-98 \%\right)$, urea (reagent grade $98 \%$ ), sodium chloride ( $\mathrm{NaCl}, 99 \%)$, magnesium chloride $\left(\mathrm{MgCl}_{2}\right.$, $98 \%)$, nitric acid $\left(\mathrm{HNO}_{3}, 70 \%\right)$ and sodium hydroxide $(\mathrm{NaOH}$, 97\%) were purchased from Sigma-Aldrich Chemie $\mathrm{GmbH}$ (Steinheim, Germany) and $\alpha$-cellulose from Sigma-Aldrich Chemie GmbH (St. Louis, USA). All chemicals were used as received.

\section{Characterization methods}

Differential scanning calorimetry (DSC) analysis of the solid GOQD powder was performed by a Mettler-Toledo DSC 820 . Around $5 \mathrm{mg}$ of sample was placed into standard $40 \mu \mathrm{l}$ aluminum cups and temperature was increased at $10{ }^{\circ} \mathrm{C} \mathrm{min}{ }^{-1}$

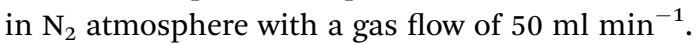

A Mettler-Toledo TGA/SDTA 851e was used for the thermogravimetric analysis (TGA) of GOQDs. Around $5 \mathrm{mg}$ of sample was placed into a $70 \mu \mathrm{l}$ alumina cup and heated at a rate of $10{ }^{\circ} \mathrm{C} \mathrm{min}$ min $^{-1}$ from $30{ }^{\circ} \mathrm{C}$ to $800{ }^{\circ} \mathrm{C}$ under an $\mathrm{N}_{2}$ flow rate of $80 \mathrm{ml} \mathrm{min}^{-1}$.

The Raman spectra of the GOQD sample was obtained using home-built spectrometer. ${ }^{44}$ The used radiation laser source (CW laser (Laser Quantum, U.K.)) had wavelength of $532 \mathrm{~nm}$.

The XPS (X-ray Photoelectron Spectroscopy) spectra were collected with a Kratos Axis Ultra DLD electron spectrometer using monochromated $\mathrm{Al} \mathrm{K} \alpha$ source operated at $150 \mathrm{~W}$. Analyser pass energy of $160 \mathrm{eV}$ for acquiring wide spectra and a pass energy of $20 \mathrm{eV}$ for individual photoelectron lines were used. The surface potential was stabilized by the spectrometer charge neutralization system. The binding energy (BE) scale was referenced to the $\mathrm{C} 1 \mathrm{~s}$ line of aliphatic carbon, set at $285.0 \mathrm{eV}$. Processing of the spectra was accomplished with the Kratos software. Powder sample for the analysis was gently handpressed into a pellet directly on a sample holder using clean Ni spatula.

The Ultraviolet-Visible (UV-Vis) absorption of GOQD solutions was measured on the instrument SHIMADZU UV-2550 UVVis spectroscopy. The band-gap of GOQDs was calculated according to eqn (1). ${ }^{45}$

$$
E=\frac{h c}{\lambda_{\text {onset }} 1.6 \times 10^{-19}}[\mathrm{eV}]
$$

Where $h$ is Planck's constant, $c$ is the speed of light and $\lambda_{\text {onset }}$ is the wavelength where the absorption has its onset according to UV-Vis spectra.

Dynamic light scattering (DLS) measurements of the GOQD solutions were carried out on Zetasizer Nano ZS from Malvern Instruments (Malvern, UK). Polylactide was used as a standard for all measurements. The scattered light was collected and measured at an angle of $173^{\circ}$ to the incident beam (non-invasive back-scattering).

Zeta potential ( $\zeta$-potential) analysis was performed using phase analysis light scattering (PALS) Zetasizer Nano ZS from Malvern Instruments (Malvern, UK) at $T=25{ }^{\circ} \mathrm{C}$.

For transmission electron microscopy (TEM), a HITACHI HT7700 instrument and the software version 02/05 were used to process all data. The GOQD solutions were drop-casted on the TEM grid (ultrathin carbon Cu grid, TED PELLA INC., USA) and the excess $\mathrm{H}_{2} \mathrm{O}$ was removed with dust free tissue after 2 min to decrease possible aggregation of nanoparticles. The samples were left to dry at dust free chamber before the analyses in highresolution mode TEM (HR-TEM).

Fluorescence spectroscopy was used for fluorescence emission measurements of GOQD solutions by utilizing Cary Eclipse Spectrophotometer from Varian. The fluorescence emission of the GOQD solutions were measured at different concentrations at the excitation wavelengths $250 \mathrm{~nm}, 350 \mathrm{~nm}$ and $450 \mathrm{~nm}$. The light emission was collected $90^{\circ}$ to the incident beam and the instrument used xenon lamp technology.

$\mathrm{pH}$-measurements of the GOQD solutions were determined by utilizing the instrument VWR symphony meter SB70P with Biotrode (Hamilton, USA). Buffer solutions with $\mathrm{pH} 4$ and $\mathrm{pH} 7$ 
were used for the calibration where the reference was a PROTELYTE electrolyte.

Atomic force spectroscopy images were acquired using a Nanoscope IIIa multimode atomic force microscopy (AFM) (Digital Instruments, Santa Barbara, CA) in tapping mode under ambient conditions $\left(T=20-25{ }^{\circ} \mathrm{C}\right)$. Aqueous solutions of GOQDs were drop-casted on the freshly cleaved mica (grade V-1, Electron Microscopy Sciences as surface) and dried under dust free conditions before measurements.

\section{Synthesis of GOQDs}

Carbon nanospheres (CNs), as new precursors for the production of GOQDs, were derived through microwave-assisted hydrothermal degradation of cellulose according to our previously reported procedure ${ }^{37}$ (see Fig. S1, ESI $\dagger$ file for the reaction scheme). As it was reported, ${ }^{46}$ GOQDs were synthesized from the cellulose based CNs by the following process: a $15 \mathrm{ml}$ solution of CNs in $70 \% \mathrm{HNO}_{3}(1: 100, \mathrm{w} / \mathrm{w})$ was put in a $100 \mathrm{ml}$ one-neck round-bottom flask and sonicated at $45{ }^{\circ} \mathrm{C}$ for 30 minutes in sonication bath. Then, the sonicated and deagglomerated CNs were heated for 60 minutes at $90{ }^{\circ} \mathrm{C}$ with magnetic stirring. $50 \mathrm{ml}$ of cold deionized $\mathrm{H}_{2} \mathrm{O}\left(15^{\circ} \mathrm{C}\right)$ was added to the final orange transparent solutions to stop the reaction and to dilute the acidic medium. Finally, acidic $\mathrm{H}_{2} \mathrm{O}$ was removed by rotary evaporation reaching an orange/red solid powder of GOQDs. The synthesized GOQDs were kept in vacuum oven (room temperature $T \sim 22{ }^{\circ} \mathrm{C}$ ) for one week to remove any remaining $\mathrm{H}_{2} \mathrm{O}$ and acid. The structure of the produced CNs and GOQDs were confirmed by multiple techniques.

\section{Results and discussions}

The 3D supramolecular self-assembly behavior of 2D GOQDs in aqueous solutions was investigated as well as the possibilities to tune the optical properties through supramolecular structures. Several techniques were applied for the analyses of GOQDs and the formed supramolecular structures including DLS, zeta potential, UV-Vis, TEM, AFM, pH measurements and fluorescence spectroscopy. The GOQD nanostructures were synthesized from $\mathrm{CN}$ bulk materials under $\mathrm{O}$ rich acidic conditions resulting in controlled oxidation-degradation reaction producing GOQD (Fig. S1, ESI $\dagger$ file). The prepared dots had a mean diameter of $1.2 \mathrm{~nm}$ and showed a GO thermal behavior that is displayed in Fig. S2, ESI $\dagger$ file. Raman spectra of the synthesized GOQDs exhibited a D-band $\left(\sim 1356 \mathrm{~cm}^{-1}\right)$ and Gband $\left(\sim 1590 \mathrm{~cm}^{-1}\right)$ usually assigned to structural disorder corresponding to functional groups such as hydroxyl, epoxy etc. and the graphitized structure, respectively. The ratio of the $\mathrm{D}$ band and G-band was $I_{\mathrm{D}} / I_{\mathrm{G}} \sim 1.08$ (Fig. S3, ESI $\dagger$ file). High resolution XPS spectrum of C1s for GOQDs exhibited sharp peaks at $284.6 \mathrm{eV}$ and $289.9 \mathrm{eV}$ corresponding to $\mathrm{C}=\mathrm{C} \& \mathrm{C}-\mathrm{C}$ bonds $(46.4 \%)$ and $\mathrm{O}=\mathrm{C}-\mathrm{O}(21.8 \%)$ bonds, respectively (Fig. 1a). Signals of $\mathrm{C}-\mathrm{O}$ at $286.0 \mathrm{eV}$ and $\mathrm{C}-\mathrm{O}-\mathrm{C}$ at $287.3 \mathrm{eV}$ displayed $23.2 \%$ and $8.6 \%$, respectively. The estimated $\mathrm{C} / \mathrm{O}$ ratio for the synthesized GOQDs was calculated to be approximately 2 . The $\mathrm{C} / \mathrm{O}$ ratio is, thus as expected slightly lower

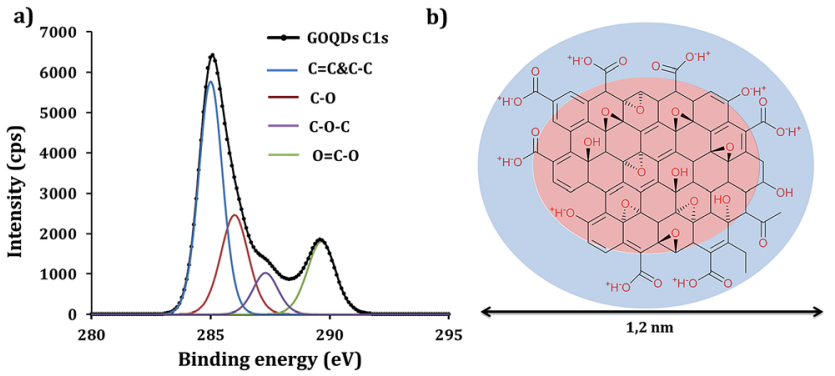

Fig. 1 (a) High resolution XPS C1s peak of the synthesized GOQDs. The position of $\mathrm{C}=\mathrm{C} \& \mathrm{C}-\mathrm{C}, \mathrm{C}-\mathrm{O}, \mathrm{C}-\mathrm{O}-\mathrm{C}$ and $\mathrm{O}=\mathrm{C}-\mathrm{O}$ is $284.6 \mathrm{eV}$, $286.0 \mathrm{eV}, 287.3 \mathrm{eV}$ and $289.9 \mathrm{eV}$, respectively. (b) Schematic representation of the GOQD structure with hydrophilic edges capable of electrostatic and hydrogen bonding interactions (light blue region) and hydrophobic basal plane capable of $\pi$-stacking, hydrogen bonding and hydrophobic interactions.

compared to values in previous studies for, graphite oxides sheets for which a ratio of 2.4 was typically reported. ${ }^{47}$ This is due to the much smaller size of the GOQDs synthesized in the present study, increasing the ratio of edges where most of the oxidized structures such as carboxylic acid are located to basal plane where the unsaturated $\mathrm{C}=\mathrm{C} \& \mathrm{C}-\mathrm{C}$ bonds are located. The placement of different functional groups at the edges and a hydrophobic basal plane enable variety of inter- and intramolecular secondary interactions in aqueous medium specifying the amphiphilic characteristics of GOQDs (Fig. 1b).

\section{Critical association concentration (CAC)}

The supramolecular self-association of the produced GOQDs was studied by utilizing several concentration dependent size measurements. As expected, the single sheet dots with diameters $\sim 1.2 \mathrm{~nm}$ were stable in the dilute regime $\left(c a . \leq 0.05 \mathrm{mg} \mathrm{ml}^{-1}\right.$ ) when the $\mathrm{pH}$ of the solution was acidic due to the ionization of the carboxylic acid functional groups, which are mainly present at the edges of the 2D dots (Table S1, ESI $\uparrow$ file). The low concentration of the GOQDs, together with the repulsive forces provided by the negative surface charge of the dots with $\zeta$ potential $=-16.3 \mathrm{mV}$ in $0.05 \mathrm{mg} \mathrm{ml}^{-1}$ solution, kept the single molecular dispersion stable (Fig. S4, ESI $\dagger$ file). Increasing the concentration of the GOQDs above $0.05 \mathrm{mg} \mathrm{ml}^{-1}$ changed the cooperativity of the systems from disfavorable to favorable regime. Supramolecularly associated dots self-assembled to the multi molecular particles with negative $\zeta$ potential $=-26.8 \mathrm{mV}$ in $0.1 \mathrm{mg} \mathrm{ml}^{-1}$ (see Fig. S4, ESI $\dagger$ file) and lower $\mathrm{pH}=4.2$ in $0.1 \mathrm{mg} \mathrm{ml}^{-1}$ solution (see Fig. 2). At concentrations higher than $0.05 \mathrm{mg} \mathrm{ml}^{-1}$, it seems that the system can energetically overcome the repulsive and disfavorable forces between the negatively charged dots making the phase separation favorable. A concentration between $0.05 \mathrm{mg} \mathrm{ml}^{-1}$ and $0.07 \mathrm{mg} \mathrm{ml}^{-1}$ can therefore be considered as the "critical association concentration" (CAC) for the GOQDs (Fig. 2). In this concentration interval the single 2D sheets of $1.2 \mathrm{~nm}$ GOQDs, found at concentrations below $0.05 \mathrm{mg} \mathrm{ml}^{-1}$, were shown to associate into larger particles of approximately $80 \mathrm{~nm}$ in diameter. 

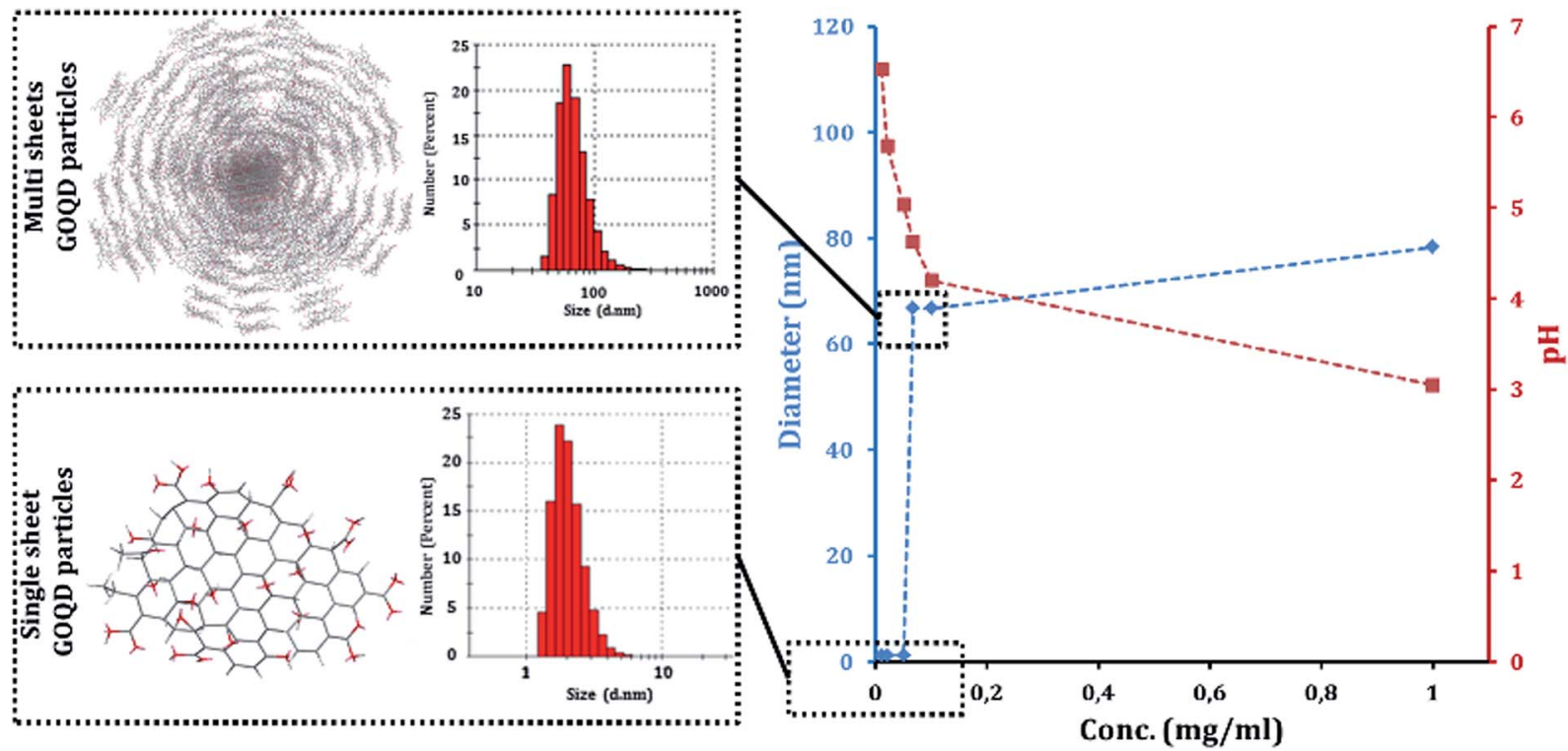

Fig. 2 Approximate size, size distribution and $\mathrm{pH}$ of the aqueous GOQD solutions as a function of concentration determined by $\mathrm{DLS}$ at $25^{\circ} \mathrm{C}$. The squares starting from the left of the figure correspond to $0.01 \mathrm{mg} \mathrm{ml}^{-1}, 0.02 \mathrm{mg} \mathrm{ml}^{-1}, 0.05 \mathrm{mg} \mathrm{ml}^{-1}, 0.067 \mathrm{mg} \mathrm{ml}^{-1} \mathrm{and} 0.1 \mathrm{mg} \mathrm{ml}^{-1} \mathrm{GOQDs}$ in deionized $\mathrm{H}_{2} \mathrm{O}$.

The morphologies and sizes of the GOQD sheets were further investigated by HR-TEM and AFM at concentrations below and above CAC (Fig. 3). The smallest recognizable sheet size that could be visualized was $\sim 4 \mathrm{~nm}$ in diameter as calculated from the HR-TEM images, which is a bit larger than the $\sim 1.2 \mathrm{~nm}$ single sheet size measured by DLS. The slightly larger size could be due to association during drying on the TEM and AFM-grids. The association of GOQDs at concentrations above CAC



Fig. 3 HR-TEM (left) and AFM (right) images of the GOQDs: (a) below $C A C$ at a concentration of 0.01 , and (b) above CAC at a concentration of $1 \mathrm{mg} \mathrm{ml}^{-1}$, showing different morphologies such as irregular spheres and 2D sheets upon aggregation. resulted in porous irregular semi-spherical particles formed by the associated and stacked dots. The self-assembly of the GOQDs seem to follow a nucleation-elongation mechanism as the concentration of the GOQDs increases. A natural characteristic of such systems, due to the week nature of the secondary interactions between the charged GOQDs, is a slow nucleation step, which further controls the initial formation of the rods and the observed structures. As the concentration of the GOQDs increased, the energetic barrier for forming stable nucleus was overcome and the nucleation was followed by rapid growth of the GOQD assemblies. Based on the observed TEM morphologies, the propagation of the formed nucleus progressed radially through addition of GOQDs, stacking on each other, forming irregular spheres by radial growth of the formed GOQD rods (Fig. 3b, for more images see Fig. S5 in the ESI $\dagger$ file).

The UV-Vis absorption of the solutions with different concentrations (both below and above CAC) exhibited two typical absorption peaks corresponding to the GO structures. ${ }^{48}$ The small peak around $320 \mathrm{~nm}$ was an $\mathrm{n} \rightarrow \pi^{*}$ transition attributed to $\mathrm{C}=\mathrm{O}$ and the peak at $200 \mathrm{~nm}$ corresponds to the $\pi \rightarrow \pi^{*}$ transition of $\mathrm{C}=\mathrm{C}\left(\lambda_{\max }\right)($ Fig. $4 \mathrm{a}$, see also a typical UVVis transmittance spectrum in Fig. S6 in ESI $\dagger$ file). The absorption was shown to increase with higher concentration in accordance to Lambert-Beers law. At the same time a hypsochromic shift (blue shift of $\lambda_{\max }=12-14 \mathrm{~nm}$ ) at the $200 \mathrm{~nm}$ transition was realized at a concentration above CAC, which indicates formation of $\mathrm{H}$-aggregates. ${ }^{4-51}$ Therefore and based on the UV-Vis, microscopy and fluorescence spectroscopy results (Fig. 8), a possible mechanism is proposed for the supramolecular self-association-assembly of the GOQDs, at the concentration above CAC, proceeding from $2 \mathrm{D}$ single sheet dispersions to 3D irregular spheres (Fig. 4b). 
a)

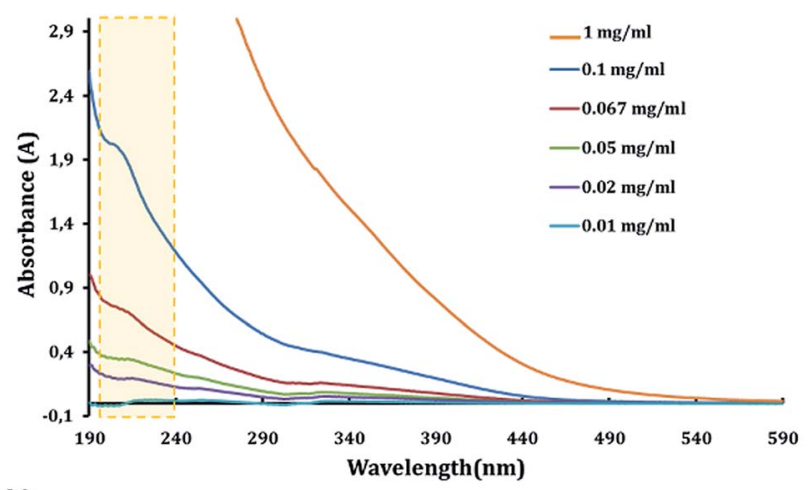

b)

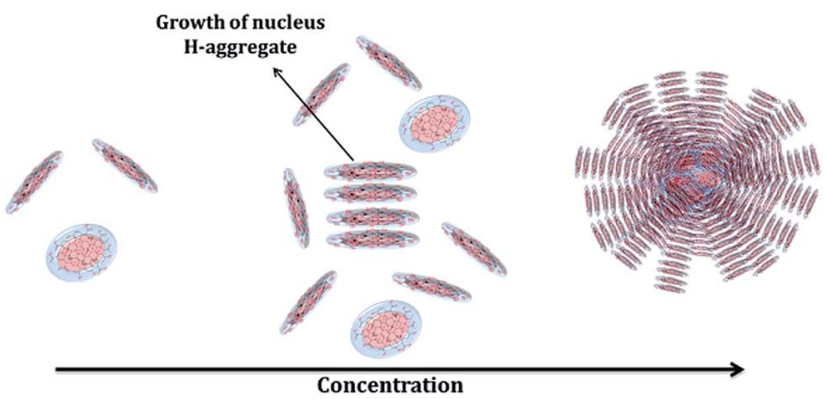

Fig. 4 (a) UV-Vis absorption of the different concentrations of GOQD solutions, (b) schematic representation of the proposed self-assembly mechanism for the GOQDs.

\section{The effect of $\mathbf{p H}$}

The proposed mechanism for the supramolecular selfassociation of the GOQDs is controlled by physical parameters of the medium and the available secondary interactions between the dots. The $\mathrm{pH}$ of the aqueous medium controls the ionization of the acidic and basic functional groups in the GOQDs and is expected to determine the association properties of the GOQD solutions.

The response of the graphene oxide structures to the $\mathrm{pH}$ of the medium is attributed to their carboxylic acid and phenolic $\mathrm{OH}$ functional groups. ${ }^{52}$ Addition of $\mathrm{HNO}_{3}$ resulted in protonation of the carboxylate groups, which were present in ionized form in the deionized water. The surface zeta potential then reached $\zeta=+5.4$, resulting in less repulsive forces between the single dots, which favored the cooperative self-assembly and formation of multi sheet particles (Fig. 5 and S7, ESI $\dagger$ file). The increased concentration of negative surface charges, ionized carboxylic and phenolic groups (COO- and $\mathrm{O}_{-}^{-}$), induced by the alkaline medium were expected to induce repulsive forces. However, opposite to what was expected the association tendency of the sheets increased at high $\mathrm{pH}$ and upon addition of $\mathrm{NaOH}$ (Fig. 5 and $\mathrm{S} 7, \mathrm{ESI} \dagger$ file). The driving force for the association in this regime seems completely different from the one observed at low $\mathrm{pH}$ and it proceeded through: (a) salting out process $^{53}$ and/or (b) hydrophobicity of the GOQDs basal plane induced by deoxygenation under the alkaline conditions ${ }^{54}$ and/ or (c) positive $\mathrm{Na}^{+}$ions originating from $\mathrm{NaOH}$, which could decrease the repulsion between the dots at a higher $\mathrm{pH}$ due to

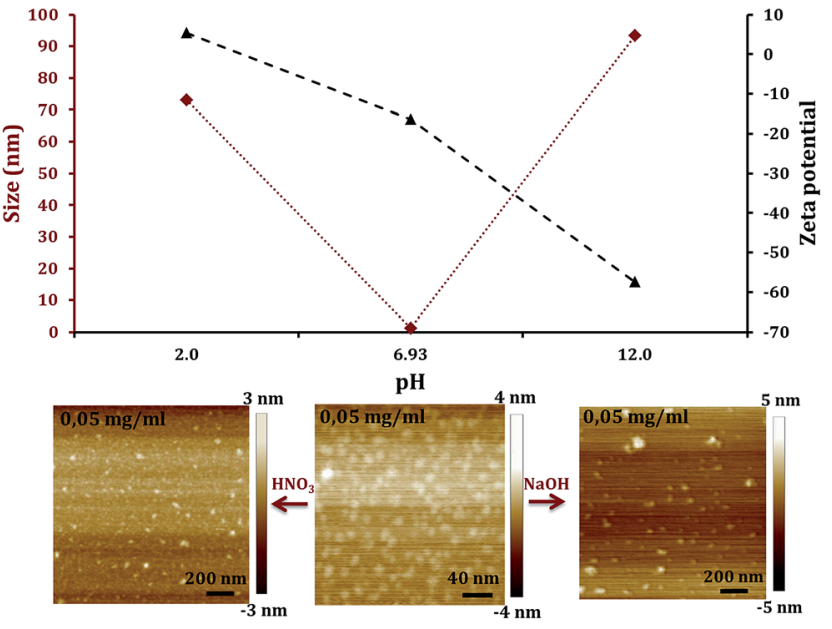

Fig. 5 Approximate size, $\zeta$-potential and AFM images of the GOGDs as a function of $\mathrm{pH}$ determined by DLS at $25^{\circ} \mathrm{C}$. In $\mathrm{HNO}_{3}$ solution $(\mathrm{pH}=$ 2.1), in pure deionized water $(\mathrm{pH}=6.9)$ and in $\mathrm{NaOH}$ solution $(\mathrm{pH}=$ 11.9) at a GOQD concentration of $0.05 \mathrm{mg} \mathrm{m}^{-1}$.

the ion-ion interactions between $\mathrm{Na}^{+}$ions and the carboxylate ions. ${ }^{55} \mathrm{~A}$ similar behavior was observed upon $\mathrm{NaCl}$ addition, see Fig. 6.

\section{The effect of different cations}

Tuning the interactions between the charged GOQDs by utilizing mono and divalent cations was also investigated (Fig. 6) by adding $\mathrm{NaCl}$ and $\mathrm{MgCl}_{2}$, respectively, to a 0.05 $\mathrm{mg} \mathrm{ml} \mathrm{m}^{-1}$ solution of the GOQDs at concentrations below CAC. The addition of divalent $\mathrm{Mg}^{2+}$ enhanced the association of the GOQDs by forming crosslinks between the sheets. $\mathrm{Mg}^{2+}$ decreased the negative $\zeta$ potential to $\zeta=-4.5$, which in turn reduced the repulsive forces and allowed GOQD associations reaching the size of $48.6 \mathrm{~nm}$. In similar fashion, addition of monovalent $\mathrm{Na}^{+}$also enhanced the association tendency between single sheets, which assembled to $65.5 \mathrm{~nm}$ diameter supramolecular particles. Interestingly, the surface charge of the particles changed to positive that could be a sign of positioning of the $\mathrm{Na}^{+}$ions at the edges of the structures. In turn, the cations decreased the repulsive forces between the negatively charged GOQDs. The capability of sodium to reduce the repulsion of the charged monomers and induce the cooperative supramolecular polymerization has previously been reported. ${ }^{56,57}$

\section{Hydrogen bonding disrupting molecules and temperature effect}

Hydrogen bonding can be considered as another possible secondary interaction between the sheets due to the presence of hydrogen donor and acceptor groups in the GOQDs chemical structure. However, as is obvious from Fig. 7a, the attempt to disrupt these interactions by addition of urea in different concentrations ${ }^{58}$ at GOQD concentration $>\mathrm{CAC}=1 \mathrm{mg} \mathrm{ml}^{-1} \mathrm{did}$ not reduce the association tendency significantly. This means that while hydrogen bonding works as one of the effective 
a)
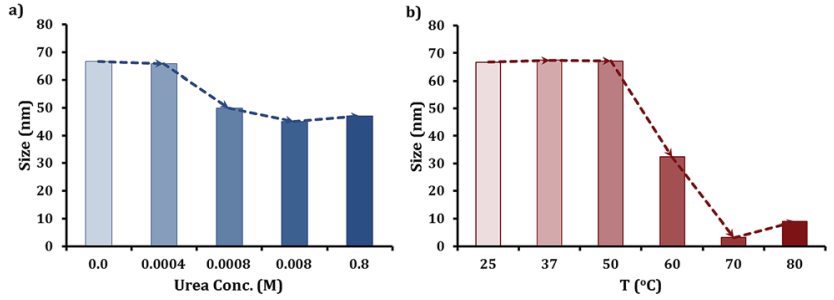

Fig. 7 Size changes in $1 \mathrm{mg} \mathrm{ml}^{-1}$ GOQD solutions at different urea concentrations (a) and temperatures (b).

secondary interactions, the main process of the self-association was controlled by electrostatic interactions between the charged dots.

The thermoresponsitivity in associated GOQD particles was also investigated in the temperatures ranging from room temperature $\left(25^{\circ} \mathrm{C}\right)$ to body temperature $\left(37^{\circ} \mathrm{C}\right)$ and finally to $80^{\circ} \mathrm{C}$. The GOQD concentration was $1 \mathrm{mg} \mathrm{ml}^{-1}$, i.e. above CAC. As is illustrated in Fig. 7b, the secondary interactions between the associated GOQD were weakened by temperatures higher than $50{ }^{\circ} \mathrm{C}$, however, some associated dots still remained stacked at $80^{\circ} \mathrm{C}(D=8.9 \mathrm{~nm})$, which is a sign of the association strength.

\section{Tunable fluorescence properties}

The semiconductive GOQD moieties are known to absorb UV and visible light of lower wavelengths. The GOQDs can based on their size, functionality, shape and fraction of $\mathrm{sp}^{2}$ to $\mathrm{sp}^{3}$, show variable fluorescence properties. ${ }^{59} \mathrm{In}$ agreement to the reported band-gap of $\mathrm{GO}^{60,61}$ the UV-Vis calculated gap from the highest occupied molecular orbital (HOMO) to the lowest unoccupied molecular orbital (LUMO) in the GOQDs was shown to be approximately $3.07 \mathrm{eV}$. In the same regime, the photoluminescence (PL) spectra of the GOQDs in selfassembled and fully exfoliated forms were shown to be completely different. As shown by Fig. 8a and b, the induced supramolecular structures from the exfoliated GOQDs at higher concentration $>\mathrm{CAC}$ or under acidic $(\mathrm{pH}=2.1)$ or basic $(\mathrm{pH}=$ 11.9) conditions induced quenching of the PL intensities using the excitation wavelengths 250 and $350 \mathrm{~nm}$ that are the wavelengths around the main structural absorbances in UV-Vis. It seems that the quenching characteristics of the $\mathrm{H}$-aggregates at the associated supramolecular structures are responsible for this observation. ${ }^{62}$ It is worth to mention that stable isotropic nematic phases were induced through similar mechanisms in the micrometer sized GO solutions at $0.5 \mathrm{wt} \%$ concentration, which is in the more concentrated regime. ${ }^{63}$ These results support the proposed association mechanism of GOQDs to supramolecular structures.

The emission peaks that appear at $700 \mathrm{~nm}$ at the excitation wavelength of $350 \mathrm{~nm}$ for the GO solutions at a concentration of $0.05 \mathrm{mg} \mathrm{ml}^{-1}$ are not attributed to the fluorescence of the GOQDs. Rather they are caused by overlap of second order emissions that are associated with the excitation wavelength. ${ }^{61,64}$

Increasing the excitation wavelength to $450 \mathrm{~nm}$ resulted in higher intensity for the $1 \mathrm{mg} \mathrm{ml}^{-1}$ solution as compared to the $0.05 \mathrm{mg} \mathrm{ml}^{-1}$ solution, but the increase was much lower than the concentration ratio. The $1 \mathrm{mg} \mathrm{ml}^{-1}$ solution was 20 times more concentrated but the maximum intensity was only 4 times higher than for the $0.05 \mathrm{mg} \mathrm{ml}^{-1}$ solution (see Fig. S8, ESI $\dagger$ file). Comparison of the emission spectra at concentrations above and below CAC for all the GOQD solutions, proved that the emission wavelength depended on the excitation wavelength, as was also shown by previous work. ${ }^{65}$ With the excitation wavelengths $250 \mathrm{~nm}, 350 \mathrm{~nm}$ and $450 \mathrm{~nm}$ for the concentrations $0.05 \mathrm{mg} \mathrm{ml}^{-1}$ and $1 \mathrm{mg} \mathrm{ml}^{-1}$, the maximum intensity in the 
a)

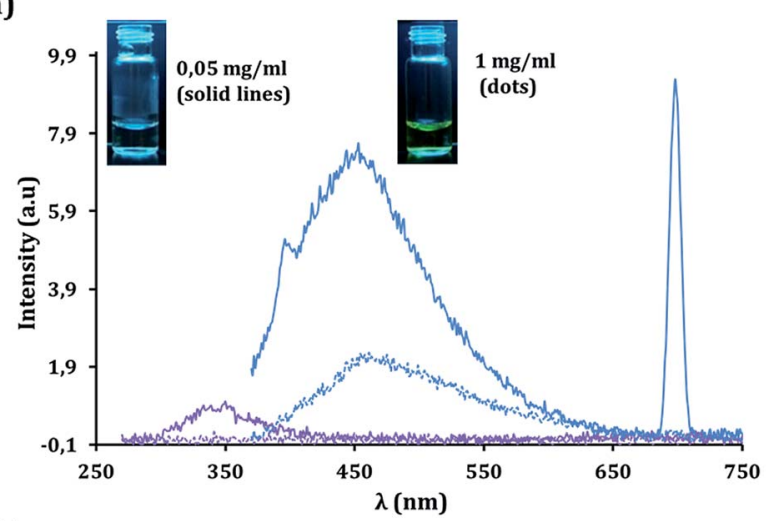

b)

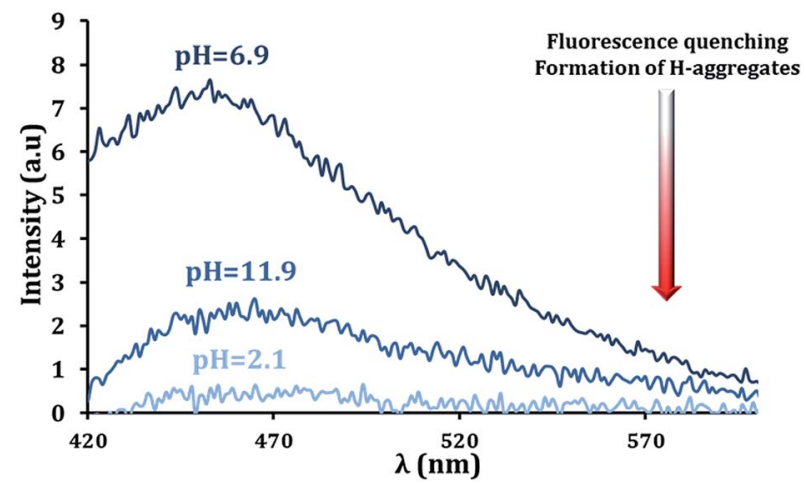

Fig. 8 (a) Fluorescence emissions of the GOQD aqueous solutions at the excitation wavelengths of $250 \mathrm{~nm}$ (violet curves) and $350 \mathrm{~nm}$ (blue curves). The visual pictures of the GOQD solutions were taken under a UV-lamp. (b) Fluorescence emissions of the $0.05 \mathrm{mg} \mathrm{ml}^{-1}$ aqueous solutions of the GOQDs at different $\mathrm{pHs}$ at the excitation wavelengths of $350 \mathrm{~nm}$.

emission spectra were $\sim 370 \mathrm{~nm}$ (violet color), $\sim 450 \mathrm{~nm}$ (blue color) and $\sim 550 \mathrm{~nm}$ (green color), respectively (see Fig. S8, ESI $\dagger$ file). Tuning the intensity and color of the emission will be possible by controlling the self-association and excitation wavelengths of the GOQD solutions.

\section{Conclusions}

The 3D supramolecular association behavior of the synthesized 2D GOQDs was systematically studied to tune the final structure and properties. The concentration dependent size measurements of the GOQDs showed a CAC between 0.05 and $0.07 \mathrm{mg}$ $\mathrm{ml}^{-1}$. The nucleation above this concentration brought the monomeric GOQDs together through cooperative self-assembly mechanism by elongation and growth of the rods, which became favourable and dominant over the repulsive forces between the negatively charged 2D single sheets. Controlling the interactions between the charged GOQDs by using mono and divalent cations $\left(\mathrm{Na}^{+}\right.$and $\left.\mathrm{Mg}^{2+}\right)$ enhanced the cooperativity, possibly through different mechanisms in each case. Deionization of the carboxylic acid-phenols at low $\mathrm{pH}(\sim 2)$ and possible salting out and/or cation addition $\left(\mathrm{Na}^{+}\right)$at high $\mathrm{pH}$ $(\sim 12)$ could decrease the repulsive forces and trigger the association between the dots. Increasing the temperature above $50{ }^{\circ} \mathrm{C}$ could weaken the secondary interactions between the associated GOQD sheets. However, the disruption of the hydrogen bonding between the GOQDs did not change the association tendency significantly. The hydrogen bonding should, thus, have limited responsibility for the association of the dots.

The fluorescence quenching and the UV-Vis blue shift of the absorption maxima $\left(12-14 \mathrm{~nm}\right.$ at $\left.\lambda_{\max } \sim 200 \mathrm{~nm}\right)$ of the supramolecular structures at a concentration $>\mathrm{CAC}$, acidic and basic $\mathrm{pH}$ and presence of cations indicated $\mathrm{H}$-aggregate association. This means that after a slow nucleation step, the elongation proceeded radially by the GOQD monomer $\mathrm{H}$ aggregate self-association, forming the final porous spheres through radial growth of the rods. The phenomenon of cooperative self-assembly - as a lesson learned from nature - in GOQDs can open new horizons from application and technological point of view for utilization of GOQDs as futuristic materials with wide application areas from electronics to biomedicine.

\section{Acknowledgements}

The Swedish Research Council (VR) is acknowledged for financial support (contract grant number 2014-4091).

\section{Notes and references}

1 A. Zandiatashbar, G.-H. Lee and S. J. An, et al., Nat. Commun., 2014, 5, 3186.

2 K. S. Novoselov, V. I. Falko, L. Colombo, P. R. Gellert, M. G. Schwab and K. A. Kim, Nature, 2012, 490, 192.

3 A. K. Geim and K. S. Novoselov, Nat. Mater., 2007, 6, 183.

4 A. K. Geim, Science, 2009, 324, 1530.

5 Y. Su and Y. Lv, RSC Adv., 2014, 4, 29324.

6 R. Kumar, S. Naqvi and N. Gupta, et al., RSC Adv., 2015, 5, 35893.

7 Z. Sun, Z. Yan, J. Yao, E. Beitler, Y. Zhu and J. M. Tour, Nature, 2010, 468, 549.

8 I. K. Moon, J. Lee, R. S. Ruoff and H. Lee, Nat. Commun., 2010, 1, 73.

9 Q. Peng and S. De, RSC Adv., 2015, 3, 24337.

10 A. Pandikumar, S. H. G. Thien and T. Peik See, et al., RSC Adv., 2014, 4, 63296.

11 W. Jie and J. Hao, Nanoscale., 2014, 6, 6346.

12 D. R. Dreyer, S. Park, C. W. Bielawski and R. S. Ruoff, Chem. Soc. Rev., 2010, 39, 228.

13 R. Rozada, J. I. Paredes and M. J. Lopez, et al., Nanoscale, 2015, 7, 2374.

14 K. Krishnamoorthy, M. Veerapandian, K. Yun and S. J. Kim, Carbon, 2013, 53, 38.

15 N. Han, T. Viet Cuong and M. Han, et al., Nat. Commun., 2013, 4, 1452.

16 Z. Wang, Y. Dong and H. Li, et al., Nat. Commun., 2014, 5, 5002 .

17 S. Stankovich, D. A. Dikin and G. H. B. Dommett, et al., Nature, 2006, 442, 282. 
18 N. Zhang, Y. Zhang and Y.-J. Xu, Nanoscale, 2012, 4, 5792.

19 Z. Liu, J. T. Robinson, X. Sun and H. Dai, J. Am. Chem. Soc., 2008, 130, 10876.

20 D. R. Dreyer, A. D. Todd and C. W. Bielawski, Chem. Soc. Rev., 2014, 43, 5288.

21 C. Galande, A. D. Mohite and A. V. Naumov, et al., Sci. Rep., 2011, 1, 85.

22 K. P. Loh, Q. Bao, G. Eda and M. Chhowalla, Nat. Chem., 2010, 2, 1015.

23 P. G. Luo, S. Sahu and S.-T. Yang, et al., J. Mater. Chem. B, 2013, 1, 2116.

24 S. N. Baker and G. A. Baker, Angew. Chem., Int. Ed., 2010, 49, 6726.

25 J. Gao, F. Bao and L. Feng, et al., RSC Adv., 2011, 1, 1737.

26 Q. Tang, Z. Zhou and Z. Chen, Nanoscale, 2013, 5, 4541.

27 Y. Xu and G. Shi, J. Mater. Chem., 2011, 21, 3311.

28 H. Y. Jeong, J. Y. Kim and J. W. Kim, et al., Nano Lett., 2010, 10, 4381.

29 D. A. Dikin, S. Stankovich and E. J. Zimney, et al., Nature, 2007, 448, 457.

30 T.-H. Kim, K.-B. Lee and J.-W. Choi, Biomaterials, 2013, 34, 8660.

31 H. Bai, C. Li, X. Wang and G. Shi, J. Phys. Chem. C, 2011, 115, 5545.

32 F. Guo, F. Kim, T. H. Han, V. B. Shenoy, J. Huang and R. H. Hurt, ACS Nano, 2011, 5, 8019.

33 C. A. Hunter and H. L. Anderson, Angew. Chem., Int. Ed., 2009, 48, 7488.

34 J. Chen, B. Yao, C. Li and G. Shi, Carbon, 2013, 64, 225.

35 V. Palermo, S. Morelli, C. Simpson, K. Mullen and P. Samori, J. Mater. Chem., 2006, 16, 266.

36 Y. Dong, J. Shao and C. Chen, et al., Carbon, 2012, 50, 4738.

37 S. Hassanzadeh, N. Aminlashgari and M. Hakkarainen, ACS Sustainable Chem. Eng., 2014, 3, 177.

38 S. Hassanzadeh, N. Aminlashgari and M. Hakkarainen, Carbohydr. Polym., 2014, 112, 448.

39 H. Bai, C. Li and G. Shi, Adv. Mater., 2011, 23, 1089.

40 A. B. Shivanandareddy, S. Krishnamurthy, V. Lakshminarayanan and S. Kumar, Chem. Commun., 2014, 50, 710.

41 L. He, J. Ye and M. Shuai, et al., Nanoscale, 2015, 7, 1616.
42 Y. Zhu, S. Murali and W. Cai, et al., Adv. Mater., 2010, 22, 3906.

43 C. Chung, Y.-K. Kim, D. Shin, S.-R. Ryoo, B. H. Hong and D.-H. Min, Acc. Chem. Res., 2013, 46, 2211.

44 E. Tyrode and F. D. J. Liljeblad, J. Phys. Chem. C, 2013, 117, 1780.

45 J. Tauc, R. Grigorovici and A. Vancu, Phys. Status Solidi, 1966, $15,627$.

46 K. H. Adolfsson, S. Hassanzadeh and M. Hakkarainen, RSC $A d v ., 2015$, 5, 26550.

47 J. Shang, L. Ma, J. Li, W. Ai, T. Yu and G. G. Gurzadyan, Sci. Rep., 2012, 2, 792.

48 R. Li, X. Liu, X. Deng, S. Zhang, Q. He and X. Chang, Mater. Lett., 2012, 76, 247.

49 F. Wurthner, Nat. Chem., 2014, 6, 171.

50 N. C. Maiti, S. Mazumdar and N. Periasamy, J. Phys. Chem. B, 1998, 102, 1528.

51 P. Jana, S. K. Maity, S. Bera, P. K. Ghorai and D. Haldar, CrystEngComm, 2013, 15, 2512.

52 D. Li, M. B. Muller, S. Gilje, R. B. Kaner and G. G. Wallace, Nat. Nanotechnol., 2008, 3, 101.

53 R. L. D. Whitby, A. Korobeinyk and V. M. Gun'ko, et al., Chem. Commun., 2011, 47, 9645.

54 X. Fan, W. Peng and Y. Li, et al., Adv. Mater., 2008, 20, 4490.

55 A. F. Metaxa, E. K. Efthimiadou, N. Boukos and G. Kordas, J. Colloid Interface Sci., 2012, 384, 198.

56 Y. He, F. Wu and X. Sun, et al., ACS Appl. Mater. Interfaces, 2013, 5, 4843.

57 C. Schaefer, I. K. Voets, A. R. A. Palmans, E. W. Meijer, P. van der Schoot and P. Besenius, ACS Macro Lett., 2012, 1, 830.

58 B. J. Bennion and V. Daggett, Proc. Natl. Acad. Sci. U. S. A., 2003, 100, 5142.

59 F. Liu, M. H. Jang, H. D. Ha, J. H. Kim, Y. H. Cho and T. S. Seo, Adv. Mater., 2013, 25, 3657.

60 F. Yang, M. Zhao, B. Zheng, D. Xiao, L. Wu and Y. Guo, J. Mater. Chem., 2012, 22, 25471.

61 K. Krishnamoorthy, R. Mohan and S. J. Kim, Appl. Phys. Lett., 2011, 98, 244101.

62 K. Liu, Y. Yao and Y. Kang, et al., Sci. Rep., 2013, 3, 2372.

63 Z. Xu and C. Gao, ACS Nano, 2011, 5, 2908.

64 S. Shukla and S. Saxena, Appl. Phys. Lett., 2011, 98, 073104. 65 S. Zhu, J. Zhang and X. Liu, et al., RSC Adv., 2012, 2, 2717. 